\title{
Research and Outlook on Northeast Asian Energy Interconnection
}

\author{
Yi Gao ${ }^{1,}{ }^{*}$, Zhe Liu ${ }^{1}$, Xinzhi Xu' ${ }^{1}$, Yanfen Guo ${ }^{1}$, Jun $L i^{1}$ \\ ${ }^{1}$ Global Energy Interconnection Development and Cooperation Organization, Beijing, China
}

\begin{abstract}
Northeast Asia is the most developed region in Asia with large energy demand, and plays an important role in the global economic development. Northeast Asia has been facing severe challenges in ensuring energy security, protecting the environment, and coping with climate change, because of their high dependency on fossil fuels and imports of oil from outside the region, and inverse distribution between energy resources and demand. In order to actively respond to climate change, promote the transition to lowcarbon energy and sustainable development in the region, achieve the grand purpose of economic prosperity, social progress and ecological protection, this study is conducted with a focus on power grid interconnection in Northeast Asia. Based on the historical energy and power data in Northeast Asia, this paper studies the development trends of energy and power demand in future by combining qualitative and quantitative methods. Considering the distribution of clean energy bases, this paper proposes an energy interconnection scheme in Northeast Asia with high clear energy penetration scenario. To form the AsiaEurope energy interconnection, the construction of the Asia-Europe interconnection channels is briefly analyzed in this paper.
\end{abstract}

\section{Research background}

Northeast Asia consists of seven main parts in six countries, namely, the Russian Far East, Northeast China, North China, Mongolia, the Democratic People's Republic of Korea (DPRK), the Republic of Korea (ROK). It is the most economically and culturally developed region in Asia and also one of the regions in the world top in economic vitality and energy demand. By 2017, the GDP of the six countries in Northeast Asia reached 20.3 trillion USD with an average annual growth rate of $5.1 \%$. It accounted for about $25 \%$ of the world total. The GDP of China and Japan was 12 trillion USD and 4.9 trillion USD, respectively, making them the world's second and third largest economies. With the continuous development of regional bilateral and multilateral cooperation, Northeast Asia is ushering in new opportunities for sustainable development.

Energy is an important material basis for economic and social development. The total primary energy demand in Northeast Asia increased from 1.7 billion tonnes of standard coal equivalent (tce) in 2000 to 2.99 billion tce in 2017 , with an average annual growth rate of $3.4 \%$. Large consumption of fossil fuels leads to a series of severe problems such as a shortage in fossil resources, environmental pollution, climate change and health issues. It is a key factor in triggering the challenge of regional sustainable development. The essense to meet these challenges and pursue sustainable development is to promote clean development. In energy sector, it is necessary to promote "clean replacement" in energy development, replacing fossil fuels with clean alternatives such as solar, wind and hydro power, and "electricity replacement" in energy consumption, replacing coal, oil and natural gas with electricity[1].

Clean energy resources in Northeast Asia are rich, but unevenly distributed. The wind energy in Northeast Asia is relatively rich with a theoretical potential of approximately $162 \mathrm{PWh} /$ year. At a height of $100 \mathrm{~m}$ from the ground, wind speed ranges from $2 \mathrm{~m} / \mathrm{s}$ to $12 \mathrm{~m} / \mathrm{s}$ [2]. There are several areas with wind speed exceeding $7 \mathrm{~m} / \mathrm{s}$ throughout the year, mainly in southern Mongolia, northern China and the Russian Far East. Solar energy resources in Northeast Asia are also abundant. The theoretical potential is $9189 \mathrm{PWh} /$ year. The global horizontal irradiance (GHI) in Northeast Asia ranges between $600 \mathrm{kWh} / \mathrm{m}^{2}$ and $1800 \mathrm{kWh} / \mathrm{m}^{2}$ [3]. The regions with GHI exceeding $1500 \mathrm{kWh} / \mathrm{m}^{2}$ include mainly southwestern Mongolia and parts of northern China. The hydropower resources in Northeast Asia are mainly distributed in the Lena and the Amur River Basins, with a technical potential of about $40 \mathrm{GW}$ and $14.5 \mathrm{GW}$, respectively. Currently, about $10 \%$ of the hydropower potential has been exploited [4].

\footnotetext{
$\overline{\text { * Corresponding author: gao-yi@geidco.org }}$
} 
In general, regional renewable energy resources are mainly concentrated in the Far East of Russia, Mongolia and the Northeast \& North China, while the main energy consumption centers are mainly located in Japan, ROK and North China. The distribution of regional energy resources and demand centers is unbalanced. Crossborder allocation of clean energy resources is one of the effective ways to improve the balance of regional energy supply and demand. Renewable energy generation outputs are intermittent, and the most efficient development and utilization way is to convert them into electricity on the site and integrated into the power grid. A wide range of interconnected power transmission network in the Northeast Asia will provide a platform for large-scale development, wide-range transmission and efficient utilization of renewable energy. The construction of the power grid interconnection in Northeast Asia has been widely concerned by many studies [5-12].

Under the overall research framework of the Global Energy Interconnection (GEI), and considering the research on the climate change, energy and power, this paper studies the development trends of power demand in future, and analyzes a development scheme in the region with energy mix dominated by clean energy to achieve the $2^{\circ} \mathrm{C}$ temperature control target of Paris Agreement. According to the development characteristics and clean energy resources endowment in Northeast Asia, the overall pattern of energy interconnection in Northeast Asia and relative cross-border transmission projects are proposed in this paper. In addition, this paper briefly analyzes the possible connection channel between Asia and Europe in the future based on the formation of Northeast Asian Energy Interconnection.

\section{Study platform and methods}

This study mainly focuses on the integrated and coordinated planning of energy and power sectors to carry out unified prediction and research. The overall framework of Global Energy Interconnection comprehensive study platform is shown in Figure 1.

In the platform, there are two key factors connecting the three fields of climate, energy and power. One is the energy emission related to climate and energy, and the other is energy converted to electricity. Based on these connection factors, the energy and power forecast results are optimized as the boundary conditions of each other, and the development trend of energy and power is predicted.

For energy demand study, "top-down" method is used to analyze the influence of economic development on energy demand from macro to micro. On the other side, "bottom-up" method is used to analyze the impact of technology progress, efficiency improvement, environmental constraints, energy policies and other factors on energy demand from micro to macro. Then, the future energy mix and energy consumption intensity are studied. The regional primary energy demand is finally analyzed by considering the future energy and power demand and the efficiency of power generation, heating, oil refining and other conversion processes.

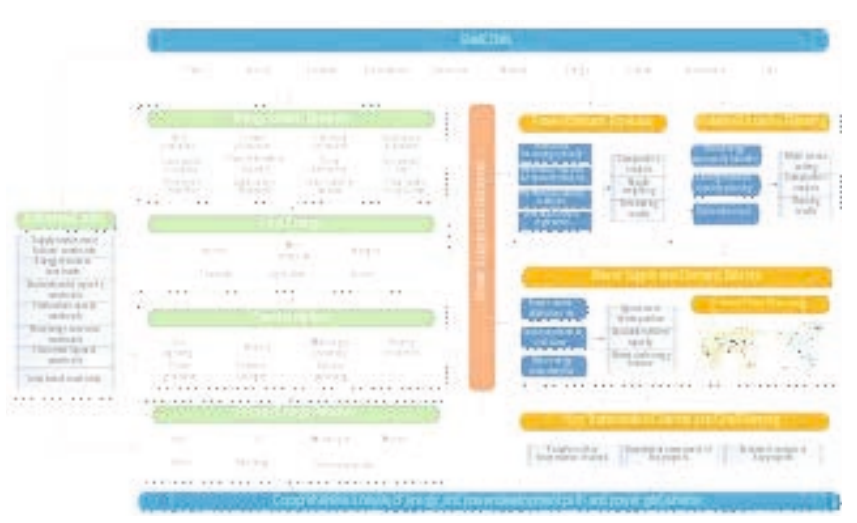

Fig. 1. Comprehensive study platform for energy and power analysis of GEI

For power demand research, the result of power demand in final energy demand obtained in previous stage is taken as a boundary condition. More analysis algorithms such as the power elasticity coefficient algorithm, the electricity consumption per capita algorithm, industry analysis method and the regression method are preferentially selected. A comprehensive load prediction results are finally calculated by weighting the prediction results under different analysis methods [13].

For power generation planning, the objective function is the minimization of total social cost including construction, operation and maintenance and fuel costs during the planning period, and constructing problems on optimization based on environmental constraints, energy resources, and power balance and to solve the planned annual power generation installed capacity, various types of installed components, timing of development, carbon emissions, etc.

On the basis of power demand forecast and power generation planning analysis, the balance analysis of power supply and demand is carried out to evaluate regional power exchange potential. Then, the network frame planning of key channels is conducted and the function of cross-border transmission channel are evaluated. Finally, the relative key projects are proposed and the investment and benefit estimation of key projects are studied.

\section{Energy and power outlook for Northeast Asia}

\subsection{Energy demand}

In a long run, the primary energy demand in Northeast Asia will first maintain flat and then slowly decline. Calculated by the partial substitution method, from 2017 to 2035, the total demand in Northeast Asia will maintain at around 3 billion tce and reach a peak of 
about 3.05 billion tce in 2025. After 2035, the total demand in Northeast Asia will begin to slowly decline. The demand will fall to 2.91 billion tce in 2050 , seeing a $3 \%$ decrease from 2017. The per capita demand in Northeast Asia will be expected to increase from 5 tce in 2017 to 5.1 tce in 2050. The forecast of primary energy demand in Northeast Asia is shown in Figure 2.

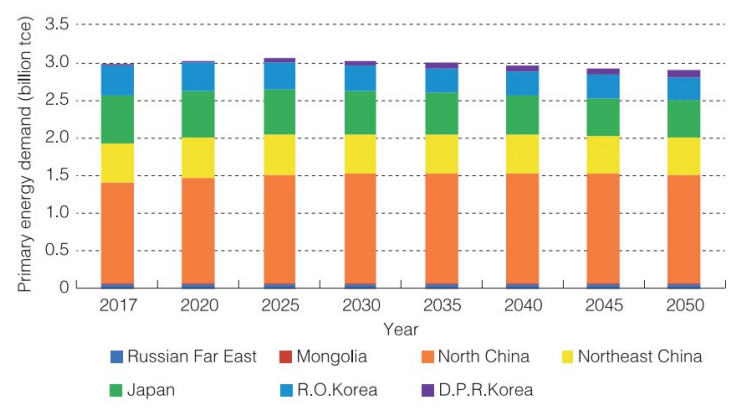

Fig. 2. Primary energy demand in Northeast Asia

From 2017 to 2025, the coal and oil demands in Northeast Asia will steadily decline, with average annual decrease rate of $2 \%$ and $1.4 \%$. The reduction in coal demand will be mainly contributed from North China, and the reduction in oil demand will be mainly contributed from Japan and the R.O.Korea. From 2025 to 2050 , the declining rate of coal and oil demands will climb to $4.6 \%$ and $2.6 \%$, respectively, and the demand in 2050 will reach 450 million tce and 320 million tce which are about $75 \%$ and $55 \%$ lower than the 2017 levels. The natural gas demand will reach a peak of about 370 million tce in around 2035, and then drop to 290 million tce in 2050, similar to the 2017 level. The demand of renewable energy such as wind and solar energy will rapidly expand with an average annual growth rate of $8 \%$, and reach 1.36 billion tce by 2050 , accounting for $51 \%$ of the total primary energy. The energy demand by fuel in Northeast Asia is shown in Figure 3.

The final energy consumption in Northeast Asia will gradually decline and the rate will accelerate after 2025 . From 2017 to 2025, the final energy consumption in Northeast Asia will slowly decline from 2.11 billion tce to 2.08 billion tce, with an average annual decrease rate of $0.2 \%$. The rate will accelerate after 2025 , making the consumption reach 1.73 billion tce in 2050. It is estimated in around 2030, electricity will overtake coal and have the highest share in the final energy.

\subsection{Power demand}

It's estimated that the power demand in the region will continue to grow. By 2025, 2035 and 2050, the electricity consumption in Northeast Asia will reach 4.5 $\mathrm{PWh}, 5.5 \mathrm{PWh}$ and $6.4 \mathrm{PWh}$, respectively. The electricity consumption in 2050 will be doubled compared with that in 2017. The annual growth rate of electricity consumption in Northeast Asia will be about
$3.7 \%$ from 2017 to $2025,2 \%$ from 2026 to 2035 , and $1 \%$ from 2036 to 2050 .

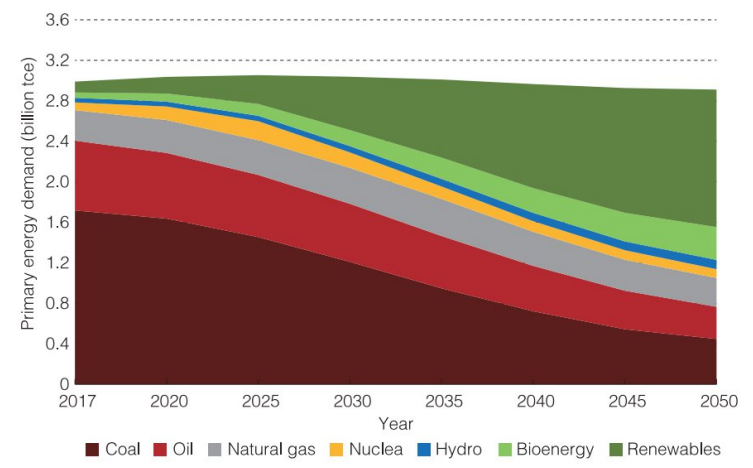

Fig. 3. Primary energy demand by fuel in Northeast Asia

North China, Northeast China, Japan and the R.O.Korea will be the major power consumers in Northeast Asia. In 2050, the electricity consumption of North China and Northeast China will be 3200 TWh and 844.9 TWh, respectively, accounting for $50 \%$ and $13 \%$ of that in Northeast Asia. The electricity consumption in Japan will reach about $1300 \mathrm{TWh}$, accounting for $21 \%$ of that in Northeast Asia. The number will be $726 \mathrm{TWh}$ in the R.O.Korea, accounting for $11 \%$ of that in the region. The D.P.R.Korea and Mongolia will consume about 184 TWh and 31.9 TWh, respectively. The power consumption in the Russian Far East will reach $88 \mathrm{TWh}$. The proportion of electricity consumption in Northeast Asia is shown in Figure 4.

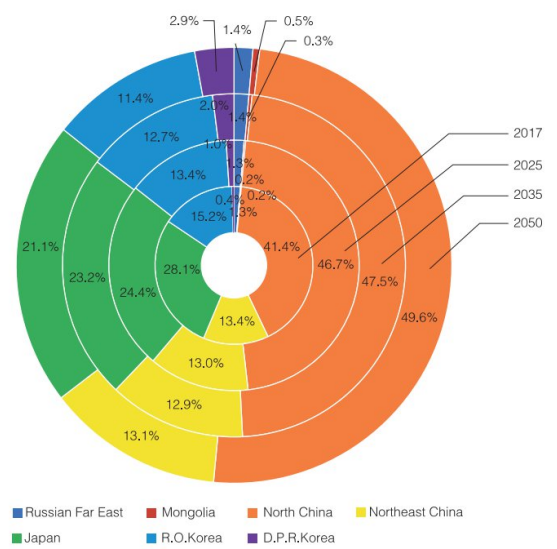

Fig. 4. Share of electricity consumption in Northeast Asia

This paper analyzes the power demand brought by electric vehicles (EVs) in the future. For passenger EVs, the development scale of passenger EV ownership is predicted through the population, per capita car ownership and proportion of EVs in the total passenger vehicles. For freight EVs, there is a close relationship between the GDP and the freight turnover in a country or a region. Therefore, the total freight turnover is predicted by assuming the total road freight turnover in the future will share the same growth rate with the GDP. For example, by 2015 , the total number of passenger vehicles in North China and Northeast China was 
approximately 54.84 million, of which EVs accounted for $0.29 \%$. The per capita car ownership was about 0.14 , and the average annual mileage was between $8000 \mathrm{~km}$ and $16000 \mathrm{~km}$. It is estimated that by 2050 , the per capita car ownership in North China and Northeast China will be 0.52 and 0.48 respectively, and the total number of passenger vehicles will reach 229 million. The proportion of EVs will increase to $40 \%-60 \%$, and thus, the annual electricity consumption for passenger EV will be 182.8- 274.2 TWh. By 2015, the total turnover of the road freight in North China and Northeast China was 4615.7 billion t-km. It is estimated that by 2050, the electrification ratio of the road freight in North China and Northeast China will reach 30\%$50 \%$, and thus, the annual electricity consumption for freight EVs will be 300.7-501.2 TWh.

\subsection{Power supply}

Based on the model of optimal planning for power generation installed capacity, the total installed capacities in 2025,2035 and 2050 are expected to reach $1.44 \mathrm{TW}, 2.05 \mathrm{TW}$ and $2.89 \mathrm{TW}$, respectively, and the increment will be $44 \%, 42 \%$ and $41 \%$ during 2017 2025, 2026 2035 and 2036 2050, respectively. The outlook for power generation installed capacity in Northeast Asia is shown in Figure 5.

With the rapid development of clean energy generation technology, the competitiveness of clean energy power generation will be significantly enhanced. The average levelized cost of electricity (LCOE) of centralized onshore wind power and photovoltaic (PV) is expected to fall to $2.4 \mathrm{US}$ cents/kWh and $1.4 \mathrm{US}$ cents $/ \mathrm{kWh}$ by 2050 , respectively. By 2035, the proportion of clean energy will surpass that of fossil energy and become the dominant power source. The installed capacity of clean energy in Northeast Asia in 2025, 2035 and 2050 will reach $0.67 \mathrm{TW}, 1.41 \mathrm{TW}$ and $2.43 \mathrm{TW}$ respectively, accounting for $47 \%, 69 \%$ and $84 \%$ of total installed capacity in Northeast Asia.

\section{Power grid interconnection}

According to the natural endowment and spatial distribution of clean energy resources in Northeast Asia, and with reference to the energy and power development plans of various countries, clean energy and power grids development require to be coordinated so as to accelerate the upgrading of national and regional power grids. Relying on advanced transmission technologies such as UHV, Northeast Asia should give full play to the regional advantages and promote the interconnection of power grids, to form a strong grid that covers clean energy bases and load centers.

Considering the power generation development, power demand distribution and the distribution of clean energy in Northeast Asia, based on the power balance analysis, North China, Japan, the R.O.Korea and the D.P.R.Korea will become the main power receiving centers. The large-scale hydro, wind and solar power plants in the Russian Far East and Mongolia will not only supply the domestic power demands but also become the main power exporting bases. The overall power flow pattern in Northeast Asia will be "West to East, North to South". The cross-border power flow within Northeast Asia will be $11.75 \mathrm{GW}, 59.75 \mathrm{GW}, 110 \mathrm{GW}$ by 2025,2035 and 2050 , respectively.

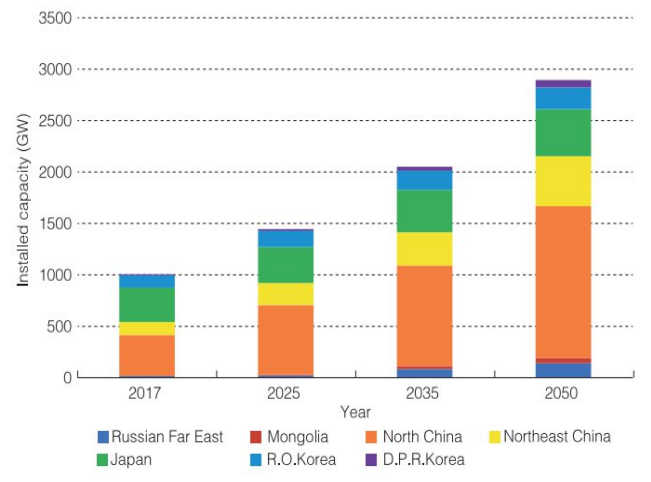

Fig. 5. Power generation installed capacity in Northeast Asia

With the development of the power grids and the large clean energy bases in Northeast Asia, through the construction of multi-directional cross-border transmission channels, clean energy electricity can be transmitted to load centers by multiple directions. A "three-ring and one-line" energy interconnection pattern will be formed by 2050, illustrated in Figure 6 .

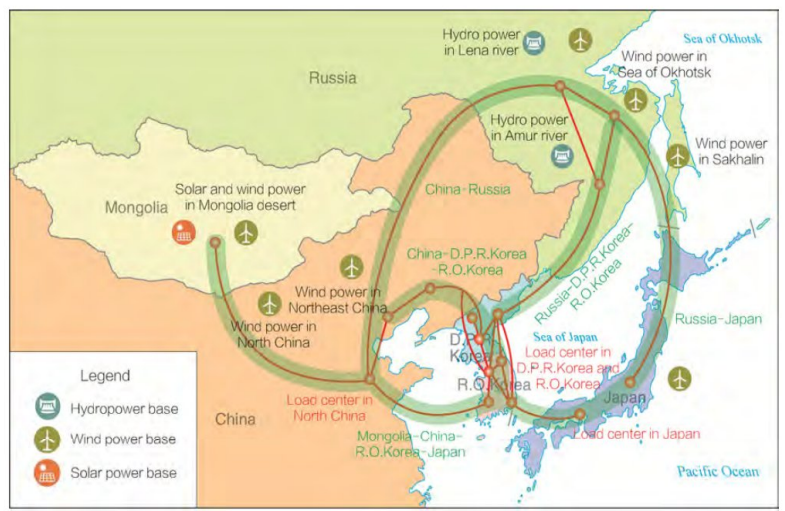

Fig. 6. Overall pattern for power grid interconnection in Northeast Asia

This interconnection mainly includes five corridors, namely, Mongolia - China - R.O.Korea - Japan, China Russia, Russia - Japan, China - D.P.R.Korea R.O.Korea and Russia - D.P.R.Korea - R.O.Korea. In the north, these corridors will connect the hydropower of the Russian Far East and the wind power in Okhotsk Sea and Sakhalin; in the west, they will connect the wind power in North China and Northeast China, as well as the solar and wind power in the Southern Mongolia; in the center, they will connect the load centers in North China, the R.O.Korea and Japan.

In order to construct the power grid interconnection in Northeast Asia, a large number of cross-border 
transmission projects are proposed and illustrated in Figure 7.

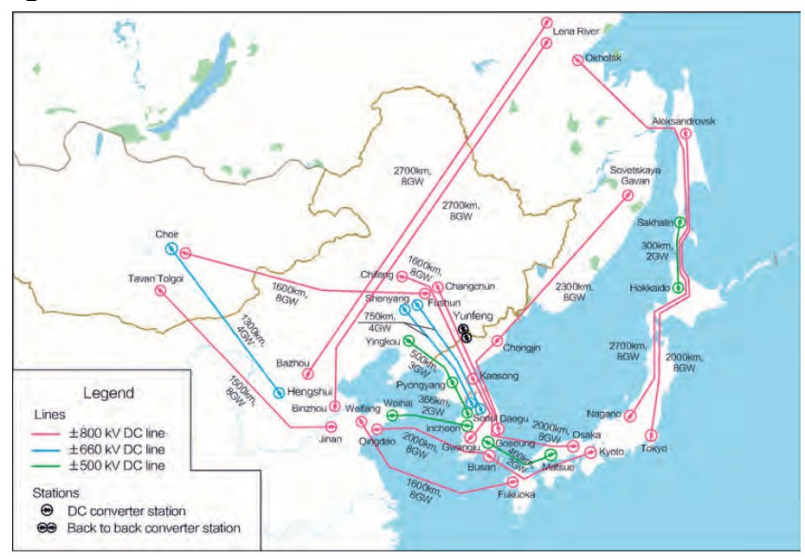

Fig. 7. Cross-border transmission projects in Northeast Asia

Northeast Asia is the most developed region in Asia with large energy demand. The Northeast Asian Energy Interconnection will play an important role in optimally allocate the energy resources and efficiently use the clean energy, deliver the sustainable development of the economy, society and environment in Asia, and promote the overall development of Asian Energy Interconnection.

\section{Asia-Europe interconnection channel prelimary analysis}

Asia is geographically connected to the Europe. AsiaEurope energy interconnection is an essential corner stone for realizing power grid interconnection between the two continents. According to the GEI research [14], the Asia-Europe connectivity channel includes the north and the south, as shown in the Figure 8.

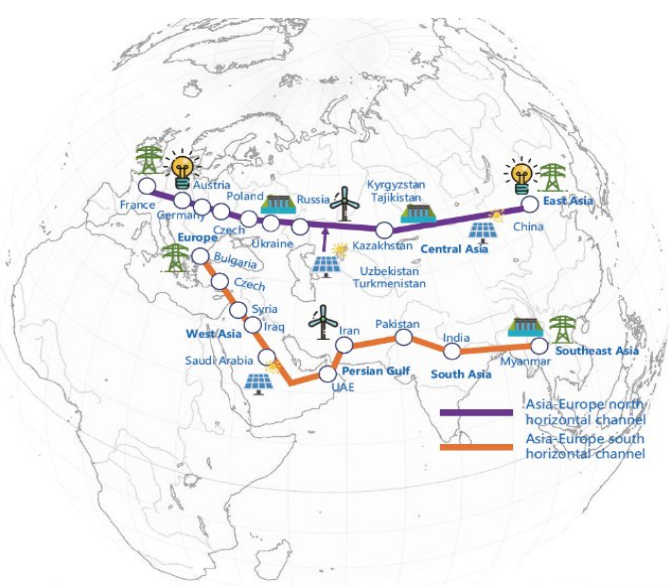

Fig. 8. Asia-Europe interconnection channels

Asia-Europe north horizontal channel will interconnect countries such as China, Kazakhstan, Germany, France, and deliver clean energy from Central Asia to Europe and China. Together with the UHV backbone grid of China, clean energy will be further delivered to Northeast Asia to provide inter-continental power support. The channel length will be about $10000 \mathrm{~km}$.
Asia-Europe south horizontal channel will interconnect Southeast Asia, South Asia, West Asia and Southern Europe, and deliver solar power from the West Asia to load centers in Southeast Europe and South Asia through the UHV DC projects. The channel will also deliver hydropower from Southeast Asia and China to South Asia. The channel length will be about $9000 \mathrm{~km}$.

Asia-Europe interconnection channels will bring the benefits of different resources, time zones and seasons. For example in China, South Asia and Europe, with a 7hour time difference at most, it will result in a diurnal load complementarity. The inter-regional and crossborder interconnection will also increase the system regulation capabilities. As shown in Figure 9, peak loads in China and South Asia are at noon in summer, and peak loads of Europe are in the evening during winter. The complementarity provided by grid interconnection between China, South Asia and Europe can reduce the peak-valley load gap by $30 \%-40 \%$.

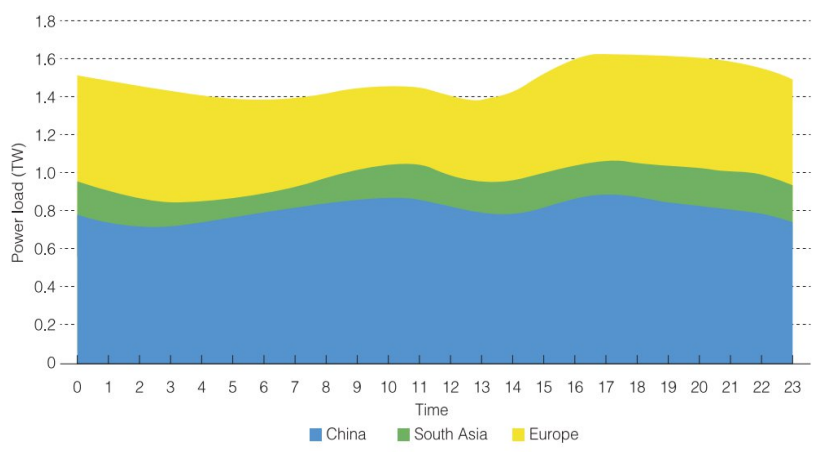

(a) Winter

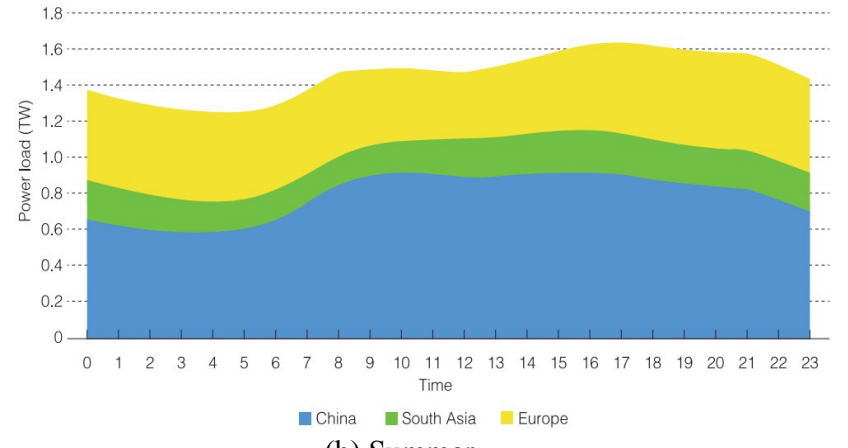

(b) Summer

Fig. 9. Typical load curves for China, South Asia and Europe

\section{Conclusions}

Under the research framework of GEI, this paper analyzes the development of energy and power in Northeast Asia in the medium and long term according to the large-scale clean energy development scenario. The study results show that the primary energy demand in Northeast Asia will reach a peak of about 3.05 billion tce in 2025 , and then slowly decline. The demand will fall to 2.91 billion tce in 2050 , seeing a $3 \%$ decrease from 2017. With the improvement of electricity replacement level in energy consumption, by 2025, 2035 and 2050, the electricity consumption in Northeast Asia 
will reach 4.5 PWh, 5.5 PWh and 6.4 PWh, respectively. The electricity consumption in 2050 will be doubled compared with that in 2017 . With the rapid decreasing of clean energy power generation cost, clean energy generation will become the dominant power source by 2035. The installed capacity of clean energy in Northeast Asia in 2025, 2035 and 2050 will reach to $0.67 \mathrm{TW}, 1.41$ TW and 2.43 TW respectively, accounting for $47 \%, 69 \%$ and $84 \%$ of total installed capacity in Northeast Asia. With reinforcing power grid interconnection between countries, a "three-ring and one-line" energy interconnection pattern will be formed by 2050 in the region. Sustainable economic, social and environmental development in Northeast Asia are expected to be promoted. Significant benefits obtained by different time zones and seasons will be achieved through the establishment of north and south interconnection channels between Asia and Europe, which will be a foundation for building the GEI.

\section{References}

1. Liu Zhenya. Global Energy Interconnection [M]. Beijing: China Electric Power Press (2015)

2. VORTEX, https://vortexfdc.com/

3. SOLARGIS, https://solargis.com/

4. Global Energy Interconnection Development and Cooperation Organization. Research and Outlook on Asian Energy Interconnection [M]. Beijing: China Electric Power Press (2019)

5. Otsuki T, Isa A B M, Samuelson R D. Electric power grid interconnections in Northeast Asia: A quantitative analysis of opportunities and challenges. Energy policy, 89, pp.311-329 (2016)

6. Energy Charter. Gobitec and Asian Super Grid for Renewable Energies in Northeast Asia [R] (2014)

7. L. S. Belyaev; L.Y. Chudinova, O. V. Khamisov, G. F. Kovalev; L. M. Lebedeva, S. V. Podkovalnikov, V. A. Savelyev, Studies of interstate electric ties in Northeast Asia. IJGEI 17 (3), pp. 228. (2002)

8. Z.Y. Liu, G.S. Chen, X.P. Guan, Q.K. Wang, W. $\mathrm{He}$, A concept discussion on Northeast Asia power grid interconnection. CSEE JPES 2 (4), pp. 87-93 (2016)

9. D. Bogdanov, C. Breyer, North-East Asian Super Grid for 100\% renewable energy supply: Optimal mix of energy technologies for electricity, gas and heat supply options. Energy Conversion and Management, 112, pp. 176-190 (2016)

10. K.H. Chung, B.H. Kim, Economic Feasibility on the Interconnected Electric Power Systems in NorthEast Asia. In Journal of Electrical Engineering and Technology, 2 (4), pp. 452-460 (2007)

11. J. Fan, X.L. Wang, Q.H. Huang, X.L. Zhang, Y. Li, P.L. Zeng, Power gird interconnection with HVDC link in Northeast Asia considering complementarity of renewable energy and time zone difference. Engineering 2019. 16, pp. 1625-1629. (2019)

12. T. Otsuki, Aishah B. M. I., R. D. Samuelson, Electric power grid interconnections in Northeast Asia_A quantitative analysis of opportunities and challenges. Energy Policy, 89, pp. 311-329, (2016)

13. H. Jiang, Y. Gao, P.F. Xu, J. Li, Study of future power interconnection scheme in ASEAN, Global Energy Interconnection, 2 (5), pp.550-560, (2019)

14. Global Energy Interconnection Development and Cooperation Organization. Research and Outlook on Global Energy Interconnection [M]. Beijing: China Electric Power Press (2019) 